\title{
Diagnóstico de contaminación atmosférica por emisiones diésel en la zona metropolitana de San Salvador y Santa Tecla
}

\author{
José Remberto Miranda-Mejía \\ Investigador Utec \\ jose.miranda@utec.edu.sv \\ Samuel Martínez-Gómez² \\ John Figerald Kenedy Hernández-Miranda ${ }^{3}$ \\ René Leonel Figueroa ${ }^{4}$ \\ Noé Aguirre 5
}

Recibido: 12/04/16 - Aceptado: 06/05/2016

\section{Resumen}

Desde hace muchos siglos la humanidad conoce algunas formas de usar el petróleo. Las emanaciones superficiales de este han sido utilizadas como fuente de luz y calor, pero su uso intensivo es relativamente moderno. El combustible diésel es un aceite ligero fraccionado del petróleo con fracciones que tienen un límite de $350^{\circ} \mathrm{C}$ en su punto de ebullición. El motor diésel es más eficiente que el de gasolina, pero por sus características es mucho más contaminante para la atmósfera. Se considera como contaminante de la atmósfera a toda sustancia ajena a composición normal atmosférica que se incorpora y permanece en ella durante un tiempo considerable.

\section{Palabras clave}

Contaminante, petróleo, combustible diésel, atmósfera

\section{Abstract}

For centuries, humanity knows some ways to use oil. Emanations of this surface have been used as a source of light and heat, but its intensive use is relatively modern. Diesel fuel is the light fractionated oil with oil fractions having a limit of $350^{\circ} \mathrm{C}$ in boiling point. The diesel engine is more efficient than gasoline, but its characteristics are much more polluting to the atmosphere. It is considered as a contaminant of the atmosphere any substance foreign to normal atmospheric composition that is incorporated and remains there for a considerable time.

\section{Keywords}

Pollution, oil, diesel fuel, atmosphere

1 Investigador Utec.

2 Docente investigador Utec.

3 Inspector de higiene y seguridad ocupacional del Ministerio de Trabajo y Previsión Social.

4 Jefe de higiene y seguridad ocupacional de Administración Nacional de Acueductos y Alcantarillados.

5 Jefe de higiene y seguridad ocupacional de Comisión Ejecutiva Portuaria Autónoma de El Salvador. 


\section{Introducción}

Las emisiones diésel en El Salvador no están reguladas, en la ciudad de San Salvador los vehículos de transporte agregan a la atmósfera gran cantidad de contaminantes. Las emisiones diésel están catalogadas como contaminantes atmosféricos con efectos cancerígenos, mutágenos y tóxicos (McClellan, 1986). El aire en San Salvador, que se respira es una de las mayores causas de infecciones respiratorias agudas (IRA), estas son la primera causa de consulta en los establecimientos de salud públicos. Hasta la semana número 23 del 2014 se habían acumulado en El Salvador 1.058 .000 consultas por esta causa.

\section{Historia de los motores diésel}

El motor diésel fue inventado por el Ing. Rudolf Diesel, quien nació en Francia. La familia de Rudolf era de origen alemán. Durante varios años, Diesel trabajó para poder utilizar otros combustibles en vez de gasolina, basados en principios de los motores de alto rendimiento térmico (ADS, 2013). La variante del motor de combustión interna, desarrollado entre los años 1893 y 1898, utilizaba el calor del aire altamente comprimido para encender el combustible inyectado en el cilindro, esto permitió duplicar la eficiencia de los demás motores de combustión interna de esa época. Cuando en 1897 Diesel presentó su motor práctico ya se estaba experimentando con motores eléctricos para locomotoras, pero la potencia de los motores diésel, como serían conocidos, fueron ganando poco a poco terreno en las líneas de largo recorrido y escaso tráfico (Gaviria, Mora, \& Agudelo, 2002). Fue empleado de la legendaria firma MAN, que por aquellos años ya producía motores y vehículos de carga (ADS, 2013).

\section{Petróleo}

Desde hace muchos siglos la humanidad conoce algunas formas de usar el petróleo. Las emanaciones superficiales de este han sido utilizadas como fuente de luz y calor, pero su uso intensivo es relativamente moderno. El petróleo se presenta en la naturaleza como un líquido color amarillo, y en algunos casos como un material semisólido de color negro. Las sustancias que componen el petróleo son fundamentalmente hidrocarburos gaseosos, líquidos y sólidos; los hidrocarburos solidos están disueltos 0 suspendidos en los líquidos (Cerutti, 2001). Las propiedades físicas y químicas de cualquier tipo de hidrocarburo o molécula dependen no solo de la cantidad de átomos de carbono en la molécula, sino también de la naturaleza de los enlaces químicos entre ellos" (The International Council on Clean Transportation, 2011).

La composición del petróleo varía mucho según los yacimientos. En general, la mayoría de los hidrocarburos son alcanos y cicloalcanos (estos se llaman naftenos en el término petroquímicos); también hay una proporción menor de aromáticos, pero, en algunos yacimientos, es mayor que el 30 \% (Yúfera, 1995).

Tabla 1. Impurezas oleofóbicas (Cerutti, 2001)

\begin{tabular}{|c|c|}
\hline Impurezas & Rango de contenido \\
\hline Sales & $10-2000 \mathrm{gr} / \mathrm{m}^{3}$ \\
\hline Agua & $0,1-5 \mathrm{Vol} . \%$ \\
\hline Sedimentos & $1-1.000 \mathrm{gr} / \mathrm{m}^{3}$ \\
\hline
\end{tabular}


Diagnóstico de contaminación atmosférica por emisiones diésel en la zona metropolitana de San Salvador y Santa Tecla José Remberto Miranda-Mejía, pp. 7-16
Revista entorno, junio 2016, número 61

ISSN: 2218-3345

Tabla 2. Impurezas oleofílicas (Cerutti, 2001)

\begin{tabular}{|c|c|}
\hline Impurezas & Rango contenido \\
\hline Compuestos de azufre & $0,1-5 \%$ en peso como S \\
\hline Compuestos órgano- metálicos & $5-400$ ppm como el metal \\
\hline Ácidos nafténicos & $0,03-0,5 \%$ en Vol. \\
\hline Compuestos de nitrógeno & $0,05-1,5 \%$ en Vol. \\
\hline
\end{tabular}

\section{Refinación del petróleo}

\section{- La destilación fraccionada del crudo}

La destilación es un proceso de separación de mezclas de líquidos. La operación fundamental de las refinerías es la destilación fraccionada del petróleo bruto para separar las fracciones con intervalos crecientes de punto de ebullición. Esto se hace en grandes calderas, con torres de rectificación muy eficaces (Yúfera, 1996). La separación de la mezcla de líquidos tiene lugar de forma que los componentes pasan a la fase gaseosa y seguidamente se condensan sin reflujo. La rectificación, Ilamada destilación en contracorriente, se aplica también a mezclas liquidas de sustancias cuyos componentes presentan pequeñas diferencias en sus temperaturas de ebullición (Hopp, 2005).

\section{Combustible Gasóleo}

El combustible diésel es un aceite ligero fraccionado del petróleo con fracciones que tienen un límite de $350^{\circ} \mathrm{C}$ en su punto de ebullición, y que contiene moléculas de entre 10 y 20 carbones, mientras que los componentes de la gasolina se ubican en el orden de 12 carbones o menos (Pemex, 1998). El combustible diésel también llamado gasóleo (gasoil), es el combustible para los motores diésel. En estos, el aire se comprime hasta $40 \mathrm{~atm}$, alcanzando temperaturas de 400 a $500{ }^{\circ} \mathrm{C}$, y en ese momento se inyecta el gasóleo líquido y pulverizado que se inflama espontáneamente. La inflamación sin retraso y sin humo es la virtud principal de un buen gasóleo. Las propiedades exigibles al diésel son contrarias a las de la gasolina; los índices de octanos (IO) son perjudiciales (Yúfera, 1996). La calidad del diésel se mide por el índice de cetano (IC). El cetano (n-hexadecano) se inflama muy bien en el motor diésel; y se le asigna un índice de cetano: $\mathrm{IC}=100$. El queroseno, el gasóleo y las gasolinas, necesitan purificación para eliminar los componentes perjudiciales, sobre todo los compuesto de azufre (S) y de nitrógeno ( $\mathrm{N}$ ), que pueden envenenar los catalizadores en los procesos de craqueo y que, en la combustión, dan azufre (S) y de nitrógeno (N) que corroen los motores y contaminan gravemente el ambiente por los tubos de escape y las chimeneas de calefacción (Yúfera, 1996).

\section{Sustancias emitidas al ambiente por combustión de motores diésel}

\section{- Formaldehído}

Este se obtiene a escala industrial por oxidación catalítica del metanol. Como catalizador se emplea el óxido de molibdeno con vestigios de hierro. El formaldehído se condensa fácilmente con fenol para dar las resinas de fenol-formaldehído que, según su grado de condensación, se emplean como plásticos y resinas sintéticas de carácter termoplástico o termoestable (Hopp, 2005). Es probable que la fuerte toxicidad del formaldehído se deba a la desnaturalización de esta proteína (albuminoide), como consecuencia de reacciones de formación de enlaces transversales (Klages, 2005).

\section{- Efectos perjudiciales}

El smog es una de las principales fuentes de exposición del formaldehído. Niveles bajos de formaldehído pueden producir irritación en la piel, los ojos, la nariz y la garganta. Gente que sufre de asma es probablemente más susceptible a los efectos de inhalación de formaldehído. Grandes cantidades de formaldehído causan profundo dolor, vómitos, coma y posiblemente la muerte (ASTDR, 1999). 


\section{- Benceno}

Es el más importante y sencillo de los hidrocarburos aromáticos, este fue descubierto en 1825 por el químico y físico brit ánico Michael Faraday, cuando investigaba la pérdida de poder iluminante del gas de hulla en los inviernos muy fríos; descubrió que el poder iluminante era producido por los vapores de una sustancia que se separaba por fraccionamiento a baja temperatura; por destilación y tratamiento con lejía se podía aislar un producto relativamente puro con reacciones con las características del benceno. La obtención del benceno se realiza exclusivamente del alquitrán de hulla (Klages, 2005).

Es un líquido incoloro con un olor dulce, se evapora muy rápidamentey se disuelve levemente en el agua, es altamente inflamable y se forma tanto en procesos naturales como en procesos industriales. Se usa para fabricar plásticos, resinas, nailon y otras fibras sintéticas, también se usa para fabricar ciertos tipos de caucho, lubricantes, tinturas, detergentes, medicamentos y pesticidas (ASTDR, 2007-A).

\section{- Efectos perjudiciales}

El benceno es un contaminante ambiental; la población en general padece exposición crónica a bajas concentraciones, siendo la más afectada la residente en las zonas de más emisión: cerca de gasolineras y de tanques de almacenamiento de combustibles y en zonas con mucho tráfico. También están expuestos los trabajadores de petroquímicas, gasolineras, aparcamientos subterráneos, talleres mecánicos y los fumadores (ASTDR, 2007-A). EI Instituto de Investigación Contra el Cáncer (IARC, por sus siglas en inglés) lo ha clasificado como carcinógeno de primera categoría: "sustancia que, por inhalación, ingestión o penetración cutánea, se sabe, es carcinógena para el hombre".

\section{Cianuro de hidrógeno}

El cianuro de hidrógeno ( $\mathrm{HCN}$ ) destaca por su gran importancia comercial, este se fabrica por oxidación catalizada por platino y rodio de una mezcla de amoniaco y metano; se utiliza principalmente para sintetizar otros compuestos, como la cianhidra de la acetona; dichos compuestos son a su vez intermedios en la producción de gomas, plásticos y fibras sintéticas (Gutsche \& Pasto, 1979).

$$
\mathrm{CH}_{4}+\mathrm{NH}_{3}+3 / 2 \mathrm{O}_{2} \longrightarrow \mathrm{HCN}+3 \mathrm{H}_{2} \mathrm{O}
$$

- Efectos perjudiciales

Es un gas incoloro y puro un líquido incoloro, es extremadamente inflamable. En caso de incendio se desprenden humos (o gases) tóxicos que son irritantes; en caso de inhalación causa confusión mental, somnolencia, dolor de cabeza náuseas, convulsiones, jadeo, pérdida del conocimiento y muerte. La sustancia se puede absorber por inhalación, a través de la piel y por ingestión (INSHT, 2003-A).

La vida media del cianuro de hidrógeno (tiempo necesario para remover la mitad del material) en la atmósfera es de aproximadamente de 1 a 3 años. El humo que se produce cuando se queman plásticos contiene cianuro, por ejemplo, poliacrilaminas, poliacrílicos, poliuretanos y otros (ASTDR, 2006).

\section{Dióxido de azufre}

La formación de dióxido de azufre (SO2) por combustión de carburantes fósiles es una fuente de contaminación. Pequeñas concentraciones de SO2 atmosférico dañan la vegetación y agravan las enfermedades respiratorias del hombre. Los materiales inertes se dañan, debido principalmente al producto ácido de la oxidación de los gases SO2 y H2S. Durante siglos en que el hombre ha conservado libros y edificaciones se ha desarrollado una importante formación de ácido sulfúrico sobre sus superficies (Spedding, 2002).

Como consecuencia, los materiales de construcción de las viejas ciudades poseen una intensa erosión, la principal fuente de contaminación por SO2 es la combustión de los carburantes fósiles, porque todos contienen compuestos de azufre como contaminantes; así, el carbón y el fuel oil contienen $3 \%$ de azufre (Spedding, 2002). Excesos de azufre son causa de destrucción porque altera el balance ecológico, esto causa la muerte de ciertas formas de vida, mientras que 
Diagnóstico de contaminación atmosférica por emisiones diésel en la zona metropolitana de San Salvador y Santa Tecla José Remberto Miranda-Mejía, pp. 7-16
Revista entorno, junio 2016, número 61

ISSN: 2218-3345 cantidades deficientes son causa de insuficiencia funcional. A escala global, el incremento de partículas de azufre en la atmósfera puede afectar el albedo de la tierra, alterando el balance de radiación (Bifani, 1999).

\section{Emisiones de motores diésel}

Las emisiones diésel están catalogadas como contaminantes atmosféricos con efectos cancerígenos, mutágenos y tóxicos; cerca de 40 sustancias han sido identificadas entre estos, anilina en las emisiones diésel (McClellan, 1986). Respirar en San Salvador ha sido muy dañino para la salud. Según el Ministerio de Medio Ambiente y Recursos Naturales (MARN), en la tarde de viernes 13 de junio a las 6:35 se midió el aire en el ambiente urbano en el cual flotaban 241 partículas, por micrómetro, del tamaño de $2.5 \mathrm{u}$. Una hora después la cantidad de partículas de hollín de 2.5 flotando en el aire era de 233 por micrómetro.

\section{Método}

\section{Tipo de estudio}

Estudio empírico con metodología cuantitativa; y descriptivo, mediante un código arbitrario de observación tipo estructurada. La investigación se llevó a cabo en el contexto habitual en el que se produce el fenómeno, pero el investigador introduce modificaciones para maximizar la probabilidad de aparición de dicho fenómeno (Montero \& León, 2007).

\section{Instrumentos}

\section{- Dräger X-act 5000}

Bomba automática para tubos Dräger que proporciona las características de flujo requeridas por los tubos de rango corto Dräger, ofrece la opción de ser utilizada con tubos y sistemas de muestreo que requieren un flujo constante. La bomba automática Dräger X-act 5000 absorbe fácilmente el aire que se debe medir a través de los tubos Dräger apropiados. La calibración de bomba se hace a través de un código de barras impreso en la etiqueta de la parte trasera de la caja de los tubos de rango corto Dräger, que contiene todos los parámetros relevantes de medición.
Simplemente pasando el código de barras por el lector de la bomba, el nombre de la sustancia que se debe medir se transfiere automáticamente a la pantalla, así como el número de emboladas y el rango de medición (Dräger, 2014).

\section{- Máscara 3M 6800}

Los respiradores 3M de la Serie 6800 están diseñados para usarse con los filtros de la serie 2000 y los cartuchos de las series 6000 y 7000 de 3M, proporcionando un alto nivel de protección en usos industriales. Las principales aplicaciones para estos respiradores son: operaciones de soldadura, industria del aluminio, industria del acero, industria del vidrio, industria farmacéutica, agroquímicos, minería, industria alimenticia, petroquímica y química, entre otras.

\section{- Cartuchos 3M 6001}

Los cartuchos $3 \mathrm{M}$ 6001, usados en la pieza facial de las series 6000 o 7000 filtro de carbón activado, que retiene vapores orgánicos.

Tubos de rango corto Dräger \# 67 28-07 benceno 5/b

Condiciones ambientales de operación del tubo Dräger \# 67 28-07 para medir benceno en aire (Dräger, 2005)

Temperatura: de 0 a $40{ }^{\circ} \mathrm{C}$

Humedad relativa: < $15 \mathrm{mg} \mathrm{H} 2 \mathrm{O} / \mathrm{L}$

Rango de aplicación del tubo \# 67 28-07

Rango de medición estándar: 5 a 50 ppm Número de carreras (emboladas de $100 \mathrm{ml}): 1$ a 20 Máximo tiempo de medición: 8 minutos aproximadamente Desviación estándar de datos: + 0 - de 10 a $15 \%$ Cambio de color: blanco $\longrightarrow$ verde marronáceo

Principio de reacción en el tubo Dräger \# 67 28-07 para medir benceno en aire (Dräger, 2005)

$\mathrm{C}_{6} \mathrm{H}_{6}+\mathrm{I}_{2} \mathrm{O}_{5}+\mathrm{H}_{2} \mathrm{SO}_{4} \longrightarrow \mathrm{I} 2+$ otras sustancias 
Tubos de rango corto Dräger para cianuro de hidrógeno 2/a 6728791

Condiciones ambientales de operación del tubo Dräger cianuro de hidrógeno 2/a 6728791 (Dräger, 2015)

Temperatura: de 0 a $30{ }^{\circ} \mathrm{C}$

Humedad relativa: < $15 \mathrm{mg} \mathrm{H} 2 \mathrm{O}$ / L

Rango de aplicación del tubo \# 672791

Rango de medición estándar: 5 a 50 ppm

Número de carreras (emboladas de $100 \mathrm{ml}$ ): 1 a 20

Máximo tiempo de medición: 8 minutos aproximadamente

Desviación estándar de datos: + o - de 10 a $15 \%$

Cambio de color: blanco $\longrightarrow$ verde marronáceo

Principio de reacción en el tubo Dräger \# 6728791 para

medir cianuro de hidrógeno en aire (Dräger, 2015)

$$
\mathrm{HCN}+\mathrm{HgCl}_{2} \longrightarrow 2 \mathrm{HCl}+\mathrm{Hg}(\mathrm{CN})_{2}
$$

\section{Tubo Dräger para medir formaldehído 2/a 8101751}

Condiciones ambientales de operación del tubo Dräger formaldehído 2/a 8101751 (Dräger, 2011-A)

Temperatura: de 5 a $40{ }^{\circ} \mathrm{C}$

Humedad relativa: $<15 \mathrm{mg} \mathrm{H} 2 \mathrm{O} / \mathrm{L}$

Rango de aplicación del tubo \# 8101751

Rango de medición estándar: 5 a 50 ppm

Número de carreras (emboladas de $100 \mathrm{ml}$ ): 1 a 20

Máximo tiempo de medición: 8 minutos aproximadamente

Desviación estándar de datos: + o - de 10 a 15 \%

Cambio de color: blanco $\longrightarrow$ verde marronáceo

Principio de reacción en el tubo Dräger \# 6728791 para medir formaldehído 2/a 8101751 en aire (Dräger, 2011-A)

$\mathrm{HCHO}+\mathrm{C}_{6} \mathrm{H}_{4}\left(\mathrm{CH}_{3}\right)_{2}+\mathrm{H}_{2} \mathrm{SO}_{4} \longrightarrow$ Productos de reacción quinoide

\section{Tubo Dräger para medir dióxido de azufre 0.5/a \# 6728491}

Condiciones ambientales de operación del tubo Dräger (Dräger, 2014-A)

Temperatura: de 5 a $40{ }^{\circ} \mathrm{C}$

Humedad relativa: $<15 \mathrm{mg} \mathrm{H} 2 \mathrm{O} / \mathrm{L}$

Rango de aplicación del tubo \# 8101751

Rango de medición estándar: 5 a 50 ppm

Número de carreras (emboladas de $100 \mathrm{ml}$ ): 1 a 20

Máximo tiempo de medición: 8 minutos aproximadamente Desviación estándar de datos: + o - de 10 a $15 \%$

Cambio de color: blanco verde marronáceo

Principio de reacción en el tubo Dräger \# 6728791 para medir dióxido de azufre 0.5/a \# 6728491 en aire (Dräger, 2014-A)

$$
\mathrm{SO}_{2}+\mathrm{I}_{2}+2 \mathrm{H}_{2} \mathrm{O} \longrightarrow \mathrm{H}_{2} \mathrm{SO}_{4}+2 \mathrm{HI}
$$

\section{Unidades de análisis}

Se evaluaron ocho lugares de San Salvador y Santa Tecla. Los lugares escogidos presentan gran cantidad de tráfico de vehículos con motores diésel, esto con el apoyo de la división de medio ambiente de la Policía (DMA) y la Sección de Seguridad Ocupacional del Ministerio de Trabajo.

\section{Resultados de medición de emisiones diésel}

Los resultados del muestreo se comparan con cada uno de sus respectivos TLV (limite umbral de exposición). En otros casos cuando no existe TLV porque la sustancia es muy peligrosa; en estos casos se ocupa STEL [límite de exposición a corto plazo, 15 minutos] (ACGIH, 2012). 
Tabla 1. Resultados de primera toma de muestra con un lugar analizado

\begin{tabular}{|c|c|c|c|c|c|c|c|}
\hline Sustancia & $\begin{array}{c}\text { Muestra } \\
\mathrm{N}^{\circ} \\
\end{array}$ & $\begin{array}{c}\mathrm{N}^{\circ} \text { de } \\
\text { emboladas }\end{array}$ & $\mathrm{mg} / \mathrm{m} 3$ & ppm & $\begin{array}{c}\text { Desviación } \\
\text { estándar } \\
+0-\end{array}$ & TLV, STEL & Condición \\
\hline $\begin{array}{c}\text { Dióxido de azufre } \\
0.5 / a\end{array}$ & 3 & 1 (reacción) & & 3 & 10 a $15 \%$ & $0.25 \mathrm{ppm}$ & Peligro \\
\hline Cianuro 2/a & 2 & 1 (reacción) & & 2 & 20 a $30 \%$ & $4.7 \mathrm{ppm}$ & No peligro \\
\hline Benceno 5/b & 3 & 5 (reacción) & & 10 & $15 \%$ & $0.5 \mathrm{ppm}$ & Peligro \\
\hline Formaldehído 2/a & 4 & $\begin{array}{c}\text { (menor que } \\
\text { 1) }\end{array}$ & & $\begin{array}{c}\text { Superó la } \\
\text { escala de } \\
40 \text { ppm }\end{array}$ & 20 a $30 \%$ & $0.3 \mathrm{ppm}$ & Peligro \\
\hline
\end{tabular}

Tabla 2. Resultados de segunda toma de muestra con un lugar analizado

\begin{tabular}{|c|c|c|c|c|c|c|c|}
\hline Sustancia & Muestra $\mathrm{N}^{\circ}$ & $\begin{array}{c}\mathrm{N}^{\circ} \text { de } \\
\text { emboladas }\end{array}$ & $\mathrm{mg} / \mathrm{m} 3$ & ppm & $\begin{array}{c}\text { Desviación } \\
\text { estándar } \\
\text { + o- }\end{array}$ & TLV, STEL & Condición \\
\hline $\begin{array}{c}\text { Dióxido de azufre } \\
0.5 / a\end{array}$ & 3 & 6 (reacción) & & 1 & 10 a $15 \%$ & $0.25 \mathrm{ppm}$ & Peligro \\
\hline Cianuro 2/a & 2 & 8 (reacción) & & 9 & 20 a $30 \%$ & $4.7 \mathrm{ppm}$ & Peligro \\
\hline Benceno 5/b & 3 & 3 (reacción) & & 2 & $15 \%$ & $0.5 \mathrm{ppm}$ & Peligro \\
\hline Formaldehído 2/a & 5 & 5 (reacción) & & $\begin{array}{c}\text { Superó la } \\
\text { escala de } \\
40 \text { ppm }\end{array}$ & 20 a $30 \%$ & $0.3 \mathrm{ppm}$ & Peligro \\
\hline
\end{tabular}


Tabla 3. Resultados de tercera toma de muestra con un lugar analizado

\begin{tabular}{|c|c|c|c|c|c|c|c|}
\hline Sustancia & Muestra $\mathrm{N}^{\circ}$ & $\begin{array}{c}\mathrm{N}^{\circ} \text { de } \\
\text { emboladas }\end{array}$ & $\mathrm{mg} / \mathrm{m} 3$ & ppm & $\begin{array}{c}\text { Desviación } \\
\text { estándar } \\
\text { + } 0 \text { - }\end{array}$ & TLV, STEL & Condición \\
\hline $\begin{array}{c}\text { Dióxido de azufre } \\
0.5 / a\end{array}$ & 3 & 11 (reacción) & & 2 & 10 a 15 & $0.25 \mathrm{ppm}$ & Peligro \\
\hline Cianuro 2/a & 2 & 10 (reacción) & & 2 & 20 a $30 \%$ & $4.7 \mathrm{ppm}$ & No peligro \\
\hline Benceno 5/b & 3 & (menor a 1) & & $\begin{array}{c}\text { Superó la } \\
\text { escala de } \\
50 \text { ppm }\end{array}$ & $15 \%$ & $0.5 \mathrm{ppm}$ & Peligro \\
\hline Formaldehído 2/a & 5 & 5 (reacción) & & 2 & 20 a $30 \%$ & $0.3 \mathrm{ppm}$ & Peligro \\
\hline
\end{tabular}

Tabla 4. Resultados de cuarta toma de muestra con un lugar analizado

\begin{tabular}{|c|c|c|c|c|c|c|c|}
\hline Sustancia & $\begin{array}{c}\text { Muestra } \\
\mathrm{N}^{\circ}\end{array}$ & $\begin{array}{c}\mathrm{N}^{\circ} \text { de } \\
\text { emboladas }\end{array}$ & $\mathrm{mg} / \mathrm{m} 3$ & ppm & $\begin{array}{l}\text { Desviación } \\
\text { estándar } \\
\text { +o - }\end{array}$ & TLV, STEL & Condición \\
\hline $\begin{array}{c}\text { Dióxido de azufre } \\
0.5 / a\end{array}$ & 3 & 5 (reacción) & & 5 & 10 a $15 \%$ & $0.25 \mathrm{ppm}$ & Peligro \\
\hline Cianuro 2/a & 2 & 5 (reacción) & & 6 & 20 a $30 \%$ & $4.7 \mathrm{ppm}$ & Peligro \\
\hline Benceno 5/b & 3 & (menor a 3) & & $\begin{array}{c}\text { Superó la } \\
\text { escala de } 50 \\
\text { ppm }\end{array}$ & $15 \%$ & $0.5 \mathrm{ppm}$ & Peligro \\
\hline Formaldehído 2/a & 5 & (menor a 3) & & $\begin{array}{c}\text { Superó la } \\
\text { escala de } 40 \\
\text { ppm }\end{array}$ & 20 a $30 \%$ & $0.3 \mathrm{ppm}$ & Peligro \\
\hline
\end{tabular}

El resultado del trabajo de campo muestra que en cuatro lugares en los que se tomó muestra, las sustancias medidas en su mayoría superaron los TLV respectivos y en algunos casos STEL, que son los tiempos de exposición de 15 minutos como máximo para algunas sustancias debido a su naturaleza muy tóxica. 
Diagnóstico de contaminación atmosférica por emisiones diésel en la zona metropolitana de San Salvador y Santa Tecla José Remberto Miranda-Mejía, pp. 7-16
Revista entorno, junio 2016, número 61

ISSN: 2218-3345

\section{Conclusiones}

- Las personas están expuestas a altos niveles de formaldehído en las emisiones de los motores diésel, ya que en la mayoría de lugares se encontraron concentraciones de hasta 133.33 veces el límite de exposición a corto plazo de 15 minutos a esta sustancia, la cual puede irritar la garganta, piel, nariz, los ojos y producir cáncer según estudios de ASTDR.

- Las personas en las paradas de buses en general están expuestas al benceno porque se encontraron altos niveles de este hidrocarburo que superan el límite umbral de exposición de $0.5 \mathrm{ppm}$ de 8 horas en una jornada laboral. Estos niveles superaban en cien veces el máximo de exposición para el ser humano.

- Las concentraciones de benceno encontradas en las mediciones son perjudiciales, ya que este entra al cuerpo por los pulmones y la piel; porque las muestras recolectadas superaron el límite de exposición a corto plazo de 15 minutos de $2.5 \mathrm{ppm}$ (STEL) en veinte veces la cantidad tolerada por el cuerpo humano. La exposición a altas concentraciones de benceno puede causar sangrados excesivos y puede afectar al sistema inmunitario, aumentando la probabilidad de contraer infecciones; también la exposición prolongada a altos niveles de benceno en el aire puede causar leucemia según ATSDR.

- Los límites de cianuro de hidrógeno encontrados en los lugares muestreados son perjudiciales porque superan los 4.7 ppm o $5 \mathrm{mg} / \mathrm{m3}$, que es el límite de exposición a corto plazo de 15 minutos en los lugares de trabajo. Las personas pueden sufrir daños en su organismo, ya que esta sustancia puede entrar por los pulmones cuando respira el aire que está contaminado con las emisiones de motores diésel según ATSDR.

- Las personas que están cerca de lugares donde transitan grades cantidades de vehículos están expuestas a concentraciones distintas de cianuro de hidrógeno.

- La exposición breve a altos niveles de cianuro puede dañar el cerebro y el corazón de las personas expuestas según estudios de ASTDR.
- El dióxido de azufre encontrado en las mediciones es muy alto y sobrepasa fácilmente, en algunos lugares muestreados, hasta veces, y en otros veinte veces el límite de exposición a corto plazo de 15 minutos STEL de $0.25 \mathrm{ppm}$. Estas concentraciones de dióxido de azufre pueden agravar las enfermedades respiratorias de las personas que están en las paradas de buses y en edificios circundantes según ATSDR, OMS y EPA.

\section{Referencias}

The international Council on Clean Transportation ICCT. (2011). "Introducción a la refinación del petróleo y producción de gasolina y Diesel con contenido ultrabajo de azufre". Recuperado de: http://www. theicct.org/sites/default/files/ICCT_RefiningTutorial_ Spanish.pdf

ACGIH (2012). ACGIH® Threshold Limit Values (TLVS $®$ ) and Biological Exposure Indices (BEls ${ }^{\circledR}$ ).. Printed the ACGIH $₫, 2012$ TLVs $®$ and BEls $\circledast$ Book. Copyright 2012.

Association of Diesel Specialists ADS (2013). "Historia de un motor en particular... el Diesel". Revista ADS, 16 de julio 2013. Recuperado de: http://diesel.org/cms/wpcontent/uploads/

ASTDR (1999). "Formaldehído". Agencia para Sustancias Tóxicas y el Registro de Enfermedades. Recuperado de http://www.atsdr.cdc.gov/es/toxfaqs/ es_tfacts111.pdf

ASTDR (2006). "Cianuro de hidrógeno". Atlanta, GA: ASTDR. Recuperado de http://www.atsdr.cdc.gov/es/toxfaqs/ es_tfacts8.pdf

ASTDR (2007-A). "Hoja informativa sobre el benceno". Atlanta, GA: ASTDR. Recuperado de: http://www. atsdr.cdc.gov/es/toxfaqs/es_tfacts3.pdf

Bifani, P. (1999). Medio ambiente y desarrollo sostenible. Madrid: Editorial IEPALA, cuarta edición.

Cerutti (2001). "La refinación del petróleo". Instituto Argentino del Petróleo y el Gas, IAPG.

Dräger (2005). Benceno. Lübeck, Germany: Drager safety AG \& Co. KGaA, 12th edition abril, 2005.

Dräger (2011). "Dräger-Tubes \& CMS Handbook". En Dräger, Dräger-Tubes \& CMS Handbook. Lübeck: Dräger. 
Dräger. (2011-A). "Formaldehído". Lübeck, Germany: Drager safety AG \& Co. KGaA,8th edition may 2011.

Dräger (2014). “Dräger X-act 5000, bomba automática para tubos Dräger". En Dräger, Dräger $X$-act 5000, bomba automática para tubos Dräger. Lübeck, Alemania.

Dräger (2014-A). "Dióxido de azufre". Lübeck: Drager safety AG \& Co. KGaA, 14th edition september 2014.

Dräger (2015). "Cianuro de hidrógeno". Lübeck, Germany: Drager safety $A G$ \& Co. KGaA,11th edition may 2015.

Gaviria, R.J.; Mora, G.J., \& Agudelo, J.R. (2002). “Historia de los motores de combustión interna". Revista Facultad de Ingenieria, $\mathrm{N}^{\circ} 26$, pp. 66-78, junio, 2002.

Gutsche, C.D., \& Pasto, D.J. (1979). Fundamentos de química orgánica. Madrid, España: Editorial Reverté, S.A., printed in Spain.

Hopp, V. (2005). Fundamentos de tecnología química. Barcelona: Editorial Reverté. Printed Publidisa, S.A de C.V.

INSHT. (2003-A). "Cianuro de hidrógeno". Madrid, España: INSHT. Recuperado de http://www.insht.es/InshtWeb/ Contenidos/Documentacion/FichasTecnicas/FISQ/ Ficheros/401a500/nspn0492.pdf

Klages, F. (2005). Tratado de química orgánica. Barcelona,
España: Editorial Reverté, tomo I (1a parte), química orgánica sistematica, impreso en España.

McClellan, R.O. (1986). “Efectos sobre la salud del Diesel: un estudio de caso en la evaluación de riesgos". En M. R. O., Efectos sobre la salud de Diesel: un estudio de caso en la evaluación de riesgos. Am Ind Hyg Assoc 47:1-13.

Montero, I., \& León, O.G. (2007 ). "A guide for naming research studies in psichology". (págs. 847-862). Madrid, España: International Journal of Clinical and Health Psychology, 2007, Vol. 7, № 3, pp 847-862.

PEMEX (1998). "El diésel". En Pemex, El diésel. Pemex. Disponible en http://www.ref.pemex.com/octanaje/24DIESEL.htm Spedding, D. (2002). Contaminación atmosférica. Barcelona: Editorial Reverté, printed in Spain.

Yúfera, E.P. (1995). "Química orgánica básica y aplicada. De la molécula a la industria". Tomo I. En E. P. Yúfera, Química orgánica básica y aplicada. De la molécula a la industria. Tomo I. Barcelona, España: Editorial Reverté, S.A.

Yúfera, E.P. (1996). Química orgánica básica y aplicada: de la molécula a la industria. Tomo I. Barcelona, España: Editorial Reverté, S.A. 